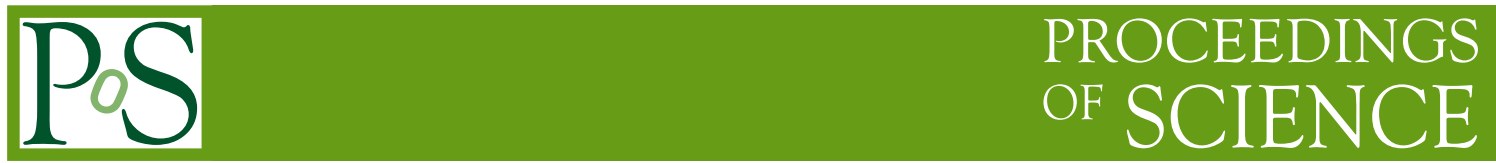

\title{
Multi-particle production in the Color Glass Condensate framework
}

\section{Cyrille Marquet*}

Centre de Physique Théorique, École Polytechnique, CNRS, 91128 Palaiseau, France

E-mail: marquetecpht.polytechnique.fr

\section{Fabio Dominguez}

Institut de Physique Théorique, CEA-Saclay, 91191 Gif-sur-Yvette, France

\section{Anna M. Stasto}

Department of Physics, Pennsylvania State University, University Park, PA 16802, USA

RIKEN BNL Research Center, Brookhaven National Laboratory, Upton, NY 11973, USA

H. Niewodniczański Institute of Nuclear Physics, Polish Academy of Sciences, Kraków, Poland

\section{Bo-Wen Xiao}

Institute of Particle Physics, Central China Normal University, Wuhan 430079, China

By studying the color structure of particle production in proton-nucleus collisions, we find that, in the large- $N_{c}$ limit, all multi-particle production processes can, up to all orders in $\alpha_{s}$, be described in terms of dipole and quadrupole scattering amplitudes only. All higher-point functions are explicitly suppressed in that limit.

XXI International Workshop on Deep-Inelastic Scattering and Related Subject-DIS2013, 22-26 April 2013

Marseille, France

\footnotetext{
*Speaker.
} 
Forward particle production in proton-nucleus collisions allows to investigate the small-x nonlinear QCD dynamics of the nuclear wavefunction with a controlled probe. Indeed, while such processes are sensitive to small-momentum (small- $x$ ) partons in the nucleus, only high-momentum partons of the proton contribute to the scattering, and their dynamics is well understood in QCD.

The Color Glass Condensate (CGC) picture of the small-x part of the nuclear wavefunction was successful in predicting $[1,2]$ - and is successful in describing $[3,4]$ - the suppression of forward particle production $[5,6]$ observed at RHIC in $\mathrm{d}+\mathrm{Au}$ compared to $\mathrm{p}+\mathrm{p}$ collisions. The same is true with the disappearance of the away-side peak of the forward di-hadron correlation function, first predicted [7], then measured [8, 9], and finally quantitatively described [10, 11, 12].

In this work, we consider multi-particle production, and show that the theoretical ingredients needed to compute single- and double-inclusive particle production, mainly the so-called dipole and quadrupole scattering amplitudes, are sufficient, in the large- $N_{c}$ limit, to also compute the cross sections for producing an arbitrary number of particles [13].

\section{High-energy eikonal scattering}

Let us start with the eikonal approximation for quarks and gluons scattering at high energies. When a dilute system of partons propagating at nearly the speed of light passes through a dense target and interacts with its strong gauge fields, the dominant couplings are eikonal: the partons have frozen transverse coordinates and the gluon fields of the target do not vary during the interaction. This is justified since the time of propagation through the target is much shorter than the natural time scale on which the target fields vary. The effect of the interaction with the target is that the partonic components of the incident wavefunction pick up eikonal phases: if $|(\alpha, x)\rangle$ (resp. $|(a, x)\rangle)$ is the wavefunction of an incoming quark of color $\alpha \in\left[1, N_{c}\right]$ (resp. gluon of color $a \in\left[1, N_{c}^{2}-1\right]$ ) and transverse position $x$ (the irrelevant degrees of freedom like spins or polarizations are not explicitly mentioned), then the action of the $S$-matrix is (see for example [14]):

$$
S|(\alpha, x)\rangle \otimes|t\rangle=\sum_{\alpha^{\prime}}\left[W_{F}(x)\right]_{\alpha \alpha^{\prime}}\left|\left(\alpha^{\prime}, x\right)\right\rangle \otimes|t\rangle, \quad S|(a, x)\rangle \otimes|t\rangle=\sum_{b} W_{A}^{a b}(x)|(b, x)\rangle \otimes|t\rangle,
$$

where $|t\rangle$ denotes the initial state of the target. The phase shifts due to the interaction are the color matrices $W_{F}$ and $W_{A}$, the eikonal Wilson lines in the fundamental and adjoint representations respectively, corresponding to propagating quarks and gluons. They are given by

$$
W_{F, A}(x)=\mathscr{P} \exp \left\{i g_{s} \int d z_{+} T_{F, A}^{a} \mathscr{A}_{-}^{a}\left(x, z_{+}\right)\right\}
$$

with $\mathscr{A}_{-}$the gauge field of the target and $T_{F, A}^{a}$ the generators of $S U\left(N_{c}\right)$ in the fundamental $(F)$ or adjoint $(A)$ representations. We use the light-cone gauge $\mathscr{A}_{+}=0$ and $\mathscr{P}$ denotes an ordering in the light-cone variable $z_{+}$along which the incoming partons are propagating.

For an incoming state $\left|\Psi_{\text {in }}\right\rangle$, the outgoing state $\left|\Psi_{\text {out }}\right\rangle=S\left|\Psi_{\text {in }}\right\rangle \otimes|t\rangle$ emerging from the eikonal interaction is obtained by the action of the $S$-matrix on the partonic components of $\left|\Psi_{\text {in }}\right\rangle$ as indicated by formula (1.1). The outgoing wavefunction $\left|\Psi_{\text {out }}\right\rangle$ is therefore a function of the Wilson lines (1.2). When calculating physical observables from $\left|\Psi_{\text {out }}\right\rangle$, one obtains objects that are target averages of traces of Wilson lines (the traces come from the color summations that one has to carry 
out). As an example, the simplest of these objects is

$$
S_{q \bar{q}}\left(x, x^{\prime}\right)=\frac{1}{N_{c}}\left\langle\operatorname{Tr}\left(W_{F}^{\dagger}\left(x^{\prime}\right) W_{F}(x)\right)\right\rangle_{t}
$$

where we have denoted the target averages $\langle t|\cdot| t\rangle=\langle\cdot\rangle_{t}$. This is the $q \bar{q}$ dipole scaterring amplitude ( $x, x^{\prime}$ : positions of the quark and antiquark) which enters for example in the $\gamma^{*}-A$ total crosssection; more generally, observables are functions of (1.3) or more complicated amplitudes. To compute these amplitudes, one has to evaluate the averages $\langle.\rangle_{t}$ which amounts to calculating averages of Wilson lines in the target wavefunction. A lot of studies are devoted to this problem, here we only study how to express multi-particle observables in dilute-dense collisions, in terms of the dipole amplitude, and potentially of higher-order correlators.

\section{Single- and double-inclusive particle production}

In the case of single-inclusive production, the $p_{\perp}$ spectrum is obtained from the Fourier transform of the dipole scattering amplitude $[15,16,17], p_{\perp}$ being conjugate to the transverse dipole size. If the produced hadron comes from the fragmentation of a quark (resp. gluon), a $q \bar{q}$ (resp. $g g$ ) dipole in involved. The fact that the exact same two-point function determines the $\gamma^{*}-A$ total cross-section and the $q+A \rightarrow h X p_{\perp}$ spectrum is profound [18].

To compute double-inclusive production, additional correlators are needed [19, 20, 21, 22]. In the large- $N_{c}$ limit, only one new object remains, the four-point function or quadrupole scattering amplitude:

$$
Q\left(x, x^{\prime}, y, y^{\prime}\right)=\frac{1}{N_{c}}\left\langle\operatorname{Tr}\left(W_{F}^{\dagger}\left(y^{\prime}\right) W_{F}(y) W_{F}^{\dagger}\left(x^{\prime}\right) W_{F}(x)\right)\right\rangle_{t} .
$$

The non-linear QCD evolution of such Wilson-line correlators with $\log (1 / x)$ is known to leading-logarithmic (LL) accuracy, at least formaly. Supplemented with running-coupling corrections, and combined with non-perturbative initial conditions (usually fitted to experimental data), the LL evolution is consistent with experimental measurements of forward hadron production and di-hadron correlations.

\section{The case of an arbitrary number of particles}

Let's assume that the cross section for the production of $\mathrm{n}$ partons is made of only dipoles and quadrupoles. Then we prove in this section that, in the large- $N_{c}$ limit, the cross section for the production of $n+1$ partons is also made of only dipoles and quadrupoles. To do this we show that, after adding a gluon line inside a dipole or a quadrupole, or between two of such objects, the leading- $N_{c}$ contributions contain only dipoles and quadrupoles. In the case of $q \bar{q}$ production (not treated explicitly in the following), one can show that the same conclusion holds when going from $\mathrm{n}-1$ to $\mathrm{n}+1$ partons. Since this is true for $n=1$ and 2 , this completes the proof.

First of all, one notices that adding a gluon line between two different objects does not increase the total number of color traces, and therefore these contributions, while they naturally involve higher-point functions, are subleading in $N_{c}$. Adding the extra gluon line within the same object will provide the leading-Nc terms. There are three such possibilities, none of them creating higherorder correlators (sextupoles, octupoles, ...): 


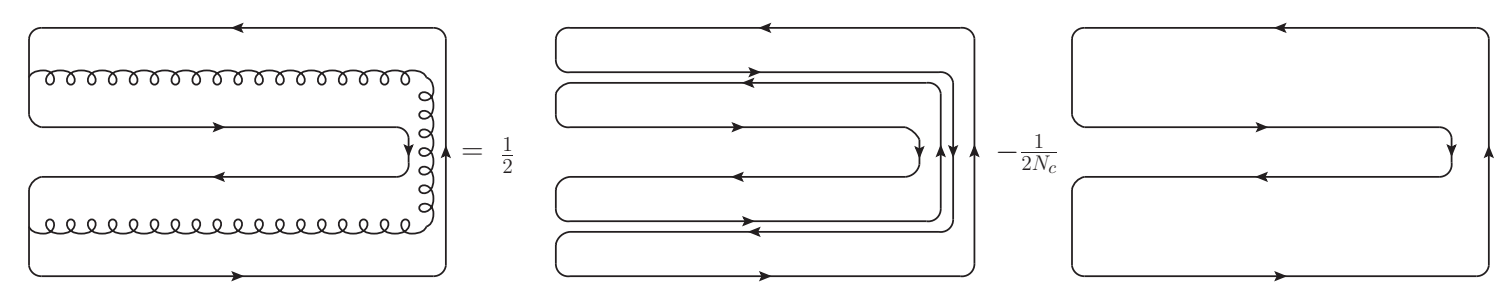

Figure 1: Diagrammatic illustration of the large- $N_{c}$ limit.

- if the extra gluon interacts both in the amplitude and in the conjugate amplitude, replacing it by a quark-antiquark double line (large- $N_{c}$ limit) adds a quadrupole to the expression for the n-parton cross section, see for example Fig. 1;

- if the extra gluon interacts only in the amplitude (or only in the conjugate amplitude), the same procedure adds a dipole to the expression for the n-parton cross section;

- finally, if the extra gluon interacts in neither the amplitude nor the conjugate amplitude, a quadrupole will split into two dipoles, and dipole will be mutiply by a factor $N_{c}$.

The proof can also be extended to include higher-order corrections. For graphs with real emissions (with additional partons going through the cut and produced - but not measured - in the final state), one can follow the above discussion, integrating over the new final-state particles does not alter the color structure. For graphs with virtual corrections, the leading-Nc terms will arise when the additional gluon is inside a dipole or inside a quadrupole. Thus higher-order virtual graphs can only generate more dipoles.

\section{Conclusions}

In conclusion, we find that in the large- $N_{c}$ limit, all multi-particle production processes in p+A-type collisions up to all orders in $\alpha_{s}$ can be described in terms of only dipoles and quadrupoles under the CGC framework. Similar conclusions were reached in [23], we have extended the result to situations where quark and gluons emissions are not eikonal. Provided we understand both dipoles and quadrupoles well, we will be able to predict any multiple-jet production processes up to corrections of order $1 / N_{c}^{2}$.

This shows the importance of understanding the evolution of the quadrupole amplitude. Several recent studies have been devoted to this issue [24, 25, 26, 27], but further work is required until the quadrupole evolution is understood as well as that of the dipole. Alternatively, a new approach was recently proposed to compute multi-particle production processes in $\mathrm{p}+\mathrm{A}$-type collisions [28], using the Langevin formuation of the non-linear QCD evolution. It applies at finite $N_{c}$, but whether such formulation is practical enough for phenomenology remains to be determined.

\section{References}

[1] D. Kharzeev, Y. V. Kovchegov and K. Tuchin, Phys. Rev. D 68, 094013 (2003). 
[2] J. L. Albacete, N. Armesto, A. Kovner, C. A. Salgado and U. A. Wiedemann, Phys. Rev. Lett. 92, 082001 (2004).

[3] A. Dumitru, A. Hayashigaki and J. Jalilian-Marian, Nucl. Phys. A 765, 464 (2006).

[4] J. L. Albacete and C. Marquet, Phys. Lett. B 687, 174 (2010).

[5] I. Arsene et al. [BRAHMS Collaboration], Phys. Rev. Lett. 93, 242303 (2004).

[6] J. Adams et al. [STAR Collaboration], Phys. Rev. Lett. 97, 152302 (2006).

[7] C. Marquet, Nucl. Phys. A 796, 41 (2007).

[8] E. Braidot for the STAR collaboration, arXiv:1005.2378.

[9] A. Adare et al. [PHENIX Collaboration], Phys. Rev. Lett. 107, 172301 (2011).

[10] J. L. Albacete and C. Marquet, Phys. Rev. Lett. 105, 162301 (2010).

[11] A. Stasto, B. -W. Xiao and F. Yuan, Phys. Lett. B 716, 430 (2012).

[12] T. Lappi and H. Mantysaari, Nucl. Phys. A 908, 51 (2013).

[13] F. Dominguez, C. Marquet, A. M. Stasto and B. -W. Xiao, Phys. Rev. D 87, no. 3, 034007 (2013).

[14] A. Kovner and U. A. Wiedemann, Phys. Rev. D 64, 114002 (2001).

[15] A. Dumitru and L. D. McLerran, Nucl. Phys. A 700, 492 (2002).

[16] Y. V. Kovchegov and K. Tuchin, Phys. Rev. D 65, 074026 (2002).

[17] C. Marquet, Nucl. Phys. B 705, 319 (2005).

[18] A. H. Mueller and S. Munier, Nucl. Phys. A 893, 43 (2012).

[19] J. Jalilian-Marian and Y. V. Kovchegov, Phys. Rev. D 70, 114017 (2004) [Erratum-ibid. D 71, 079901 (2005)].

[20] N.N. Nikolaev, W. Schafer, B.G. Zakharov and V.R. Zoller, Phys. Rev. D 72, 034033 (2005).

[21] R. Baier, A. Kovner, M. Nardi and U. A. Wiedemann, Phys. Rev. D 72, 094013 (2005).

[22] F. Dominguez, C. Marquet, B. -W. Xiao and F. Yuan, Phys. Rev. D 83, 105005 (2011).

[23] A. Kovner and M. Lublinsky, JHEP 0611, 083 (2006).

[24] A. Dumitru and J. Jalilian-Marian, Phys. Rev. D 82, 074023 (2010).

[25] F. Dominguez, A. H. Mueller, S. Munier and B. W. Xiao, Phys. Lett. B 705, 106 (2011).

[26] A. Dumitru, J. Jalilian-Marian, T. Lappi, B. Schenke and R. Venugopalan, Phys. Lett. B 706, 219 (2011).

[27] E. Iancu and D. N. Triantafyllopoulos, JHEP 1111, 105 (2011); JHEP 1204, 025 (2012).

[28] E. Iancu and D. N. Triantafyllopoulos, arXiv:1307.1559 [hep-ph]. 